\title{
Efecto del Procedimiento de Soldadura por FSW en la Evolución Temporal de las Propiedades en Uniones de AA 7075-T651
}

\section{(Effect of FSW Procedure on the Time-Evolution of the Properties in AA 7075-T651 Joints)}

\author{
Leonardo N. TUFARO', Hernán G. SVOBODA ${ }^{2,3}$ \\ ${ }^{1}$ Instituto Nacional de Tecnología Industrial, Centro de Investigación y Desarrollo en Mecánica, San Martín, Buenos Aires, Argentina. \\ ltufaro@inti.gob.ar \\ ${ }^{2}$ Universidad de Buenos Aires, Laboratorio de Materiales y Estructuras, INTECIN, Facultad de Ingeniería, Ciudad de Buenos Aires, \\ Argentina.hsvobod@fi.uba.ar \\ ${ }^{3}$ Consejo Nacional de Investigaciones Cientificas y Técnicas, Ciudad de Buenos Aires, Argentina.
}

\section{Resumen}

Se estudió el efecto del procedimiento de soldadura sobre la evolución temporal de las propiedades mecánicas de uniones soldadas por fricción-agitación (FSW) en chapa de AA7075-T651. A este fin se realizaron soldaduras por FSW bajo distintas condiciones de soldadura (velocidad de rotación y velocidad de avance) de modo de variar el aporte térmico. Se registraron los ciclos térmicos mediante la instrumentación de termocuplas y se determinó el aporte térmico neto en cada caso. Las uniones soldadas fueron caracterizadas macroestructuralmente, se determinaron perfiles de microdureza Vickers y la resistencia a la tracción a distintos tiempos luego de realizada la soldadura, los cuales variaron entre 1 y 900 días. Se observó que la dureza mínima $\left(H V_{\text {min }}\right.$ ) se ubicó en la zona afectada térmicamente (HAZ) y que la misma aumentó al disminuir el aporte térmico introducido durante la soldadura. Asimismo, dicho valor $H V_{\text {min }}$ aumentó con el tiempo, siguiendo una evolución logarítmica para todas las condiciones de soldadura. Este aumento fue mayor para la probeta soldada con menor aporte térmico. A su vez, la resistencia a la tracción de las uniones soldadas aumentó con el tiempo, evolucionando también en forma logarítmica, alcanzando una eficiencia de junta del $80 \%$.

Palabras-clave: FSW; aluminio; AA 7075-T651; termoenvejecible; propiedades mecánicas.

Abstract: It was studied the effect of the welding procedure on the temporal evolution of the mechanical properties in AA 7075-T651 friction stir welded joints. To vary the heat input samples with different parameters (rotation and welding speed) were welded. Thermal cycles were acquired by means of thermocouples instrumented on the samples, obtaining the heat input for each case. The welded joints were macrostructural characterized using optical microscopy. Vickers microhardness profiles and tensile tests were measured for different times after welding (between land 900 days). The minimum hardness $\left(H V_{\text {min }}\right.$ ) value was located in the heat affected zone (HAZ). That $H V_{\text {min }}$ decreased with heat input and was increased with the time elapsed after welding showing a logarithmic relationship, for the different welding conditions analyzed. This $H V_{\text {min }}$ increasing was higher for the sample welded with the lower heat input. Tensile strength of welded joints increased with the time after welding, according to a logarithmic relation too, reaching an joint efficiency of $80 \%$.

Key-words: FSW; aluminum; AA 7075-T651; strain aging; mechanical properties.

\section{Introducción}

La Soldadura por Fricción-Agitación (FSW) ha sido un campo de fuerte desarrollo en los últimos años. En particular la introducción de este proceso ha revolucionado la construcción de estructuras soldadas de aleaciones de aluminio, encontrando gran cantidad de aplicaciones en industrias como aeronáutica, aeroespacial, automotriz y naval, entre otras. La aleación AA7075-T651 es una aleación de alta resistencia del sistema Al-Zn-Mg-Cu, de gran aplicación en uso aeronáutico, que no es

Recebido em 22/04/2013, texto final em 05/01/2015.

DOI: http://dx.doi.org/10.1590/0104-9224/SI1904.06 soldable por procesos de fusión, siendo en la actualidad soldada por FSW. Esta aleación es del tipo termotratable y se entrega en condición de máxima dureza, alcanzando los $550 \mathrm{MPa}$ de resistencia a la tracción [1].

En aleaciones de aluminio termotratables, el ciclo térmico desarrollado durante la soldadura afecta la microestructura original del material base, debido a fenómenos de disolución, precipitación y sobreenvejecido. En las zonas alejadas a la fuente de calor los precipitados aumentan su tamaño y se reducen en número, mientras que a medida que disminuye la distancia a la línea central aumenta la temperatura pico y la disolución de los mismos. Esto produce que la dureza disminuya desde un valor máximo, que es la dureza del material base, hasta un valor mínimo a medida que disminuye la distancia a la línea de soldadura. 
Luego, existe una re-precipitación durante el enfriamiento que produce un aumento en la microdureza en las cercanías de la zona agitada (WN). De esta manera, los perfiles de microdureza obtenidos para aleaciones de aluminio termoenvejecibles tienen una distribución con forma de "W" con un valor mínimo en la zona afectada por el calor, que se debe a la competencia entre la disolución y la re-precipitación [2]. Asimismo, la resistencia a la tracción de la unión soldada queda definida por dicha zona de dureza mínima, estableciéndose una eficiencia de junta, en general, menor que la unidad [3].

Debido a los fenómenos mencionados anteriormente, luego de realizada la soldadura tiene lugar un envejecimiento natural, variando las propiedades de las uniones soldadas en estas aleaciones con el tiempo posterior a soldadura. En particular, se han reportado variaciones en las propiedades mecánicas de las uniones soldadas por fricción-agitación de AA 7075-T651 luego de soldada. Se ha observado un aumento significativo de la dureza en las distintas zonas de la unión en tiempos de envejecimiento de hasta 2300 días aproximadamente y un incremento continuo de la resistencia a la tracción, alcanzando un valor del $29 \%$ a los 3000 días aproximadamente luego de la soldadura $[4,5]$. Sin embargo, es escasa la información sistemática disponible respecto del efecto del procedimiento de soldadura sobre dicha evolución temporal de las propiedades. Por lo tanto, es de interés lograr una mayor comprensión de la evolución de las propiedades mecánicas como consecuencia del envejecimiento natural luego de la soldadura.

El objetivo del presente trabajo es estudiar el efecto del procedimiento de soldadura sobre la evolución temporal de las propiedades mecánicas de uniones soldadas por FSW en chapa de AA7075-T651.

\section{Materiales y Métodos}

\subsection{Material}

En el presente trabajo se utilizó chapa de $4 \mathrm{~mm}$ de espesor de la aleación de aluminio AA 7075-T651. Para el estudio de

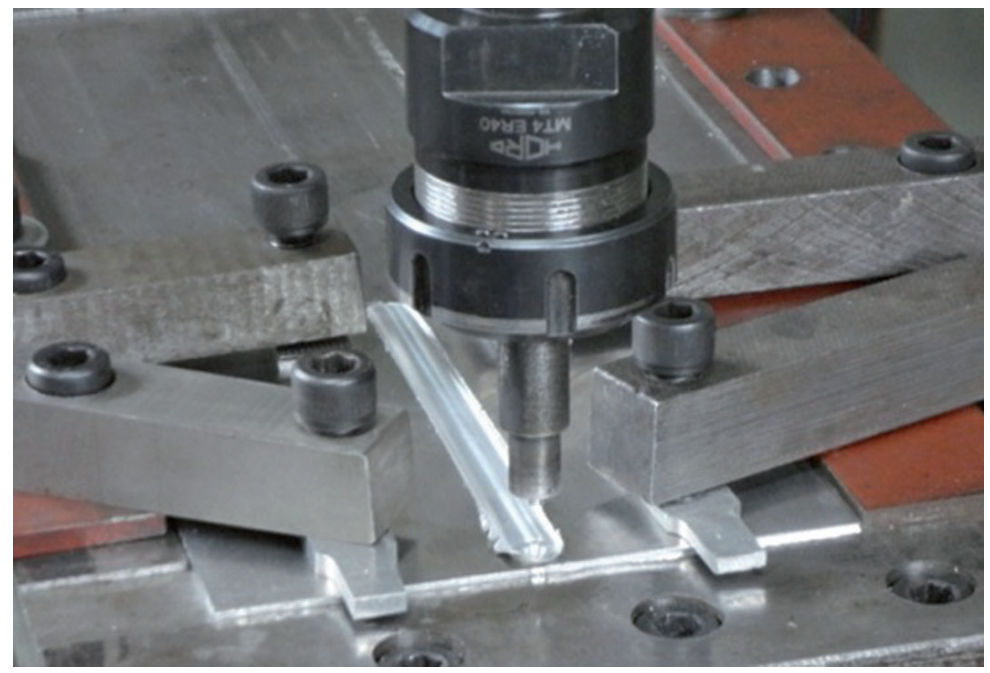

A

Figura 1. A. Soldadura por fricción-agitación (FSW), B. Ubicación de las TCs para la adquisición de ciclos térmicos los ciclos térmicos durante FSW se considera una densidad (r) de $2800 \mathrm{~kg} \cdot \mathrm{m}^{-3}$, una conductividad térmica $(\mathrm{k})$ de $130 \mathrm{~W} \cdot \mathrm{m}^{-1} \mathrm{y}$ un calor específico (C) de $960 \mathrm{~J}\left(\mathrm{~kg} .{ }^{\circ} \mathrm{K}\right)^{-1}[6]$. Sobre el material base se determinó la microdureza Vickers (HV500g) y las propiedades en tracción, siguiendo los lineamientos de la norma ASTM E8M.

\subsection{Ejecución de Uniones Soldadas y Adquisición de Ciclos Térmicos}

Se soldaron con el proceso FSW probetas de AA7075-T651 de $150 \times 75 \times 4 \mathrm{~mm}$ a tope, bajo distintas condiciones operativas, utilizando una máquina herramienta adaptada. En la Tabla 1 se muestran los parámetros empleados para las tres condiciones de soldadura utilizadas. La velocidad de rotación (w) y la velocidad de avance (U) se variaron de manera tal de obtener diferentes números de Pitch y calores aportados, mientras que se mantuvo invariante el ángulo de inclinación de la herramienta.

Tabla 1. Parámetros de soldadura por FSW y designación de probetas.

\begin{tabular}{|c|c|c|c|c|}
\hline Designación & $\begin{array}{c}\text { Velocidad de } \\
\text { Rotación } \omega \\
(\mathrm{rpm})\end{array}$ & $\begin{array}{c}\text { Velocidad } \\
\text { de } \\
\text { Avance U } \\
\left(\mathrm{mm}^{\mathrm{min}} \mathrm{m}^{-1}\right)\end{array}$ & $\begin{array}{c}\text { Ángulo de } \\
\text { Inclinación } \\
\left({ }^{\circ}\right)\end{array}$ & $\begin{array}{c}\text { Pitch }= \\
\mathrm{w} / \mathrm{U} \\
\left(\mathrm{rev} \cdot \mathrm{mm}^{-1}\right)\end{array}$ \\
\hline $514-206$ & 514 & 206 & & 2,5 \\
\hline $514-73$ & 514 & 73 & \multirow{2}{*}{2,0} & 7,0 \\
\hline $680-51$ & 680 & 51 & & 13,3 \\
\hline
\end{tabular}

La herramienta utilizada presentó un pin cónico, un hombro cóncavo y fue construida en acero para herramientas del tipo H13. El diámetro del hombro es de $12 \mathrm{~mm}$ y los diámetros mayor y menor del pin son de 4 y $3 \mathrm{~mm}$, respectivamente. Por último, la longitud del pin es de $3,8 \mathrm{~mm}$.

La medición de ciclos térmicos durante la soldadura se realizó con tres termocuplas tipo $\mathrm{K}$, las que se ubicaron en la mitad de la longitud de la probeta, en el lado de retroceso. Las

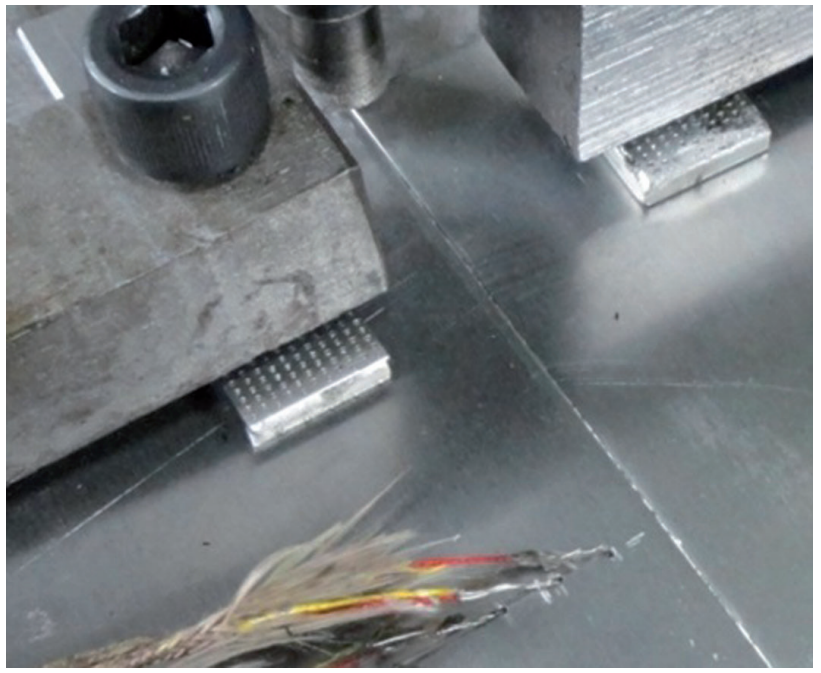

B 
mismas se posicionaron a diferentes distancias de la línea de la soldadura (TC1, TC2 y TC3, respectivamente), colocándose en orificios de $1 \mathrm{~mm}$ de diámetro y $2 \mathrm{~mm}$ de profundidad. En la Figura 1.A se observa el sistema de soldadura por FSW y una probeta soldada por el mismo, mientras que en la Figura 1.B se muestra la posición de las termocuplas respecto a la línea de soldadura (TCs).

\subsection{Modelado por Elementos Finitos (FEM) de la Transferencia de Calor}

Para obtener los valores netos de la potencia (Q) y el calor aportado $(\mathrm{H})$ a partir de los ciclos térmicos adquiridos, se modeló por elementos finitos (FEM) la transferencia de calor durante el proceso de soldadura. Para ello, se consideró que la transferencia de calor es bidimensional y solo por conducción en las chapas a soldar, resolviendo el modelo en forma transitoria [7].

Asimismo, se utilizó una distribución espacial de la fuente de calor, lineal con la distancia al centro de la misma. Esto se debe a que la densidad del calor generado por la fricción entre el hombro de la herramienta y las chapas a soldar aumenta con la velocidad tangencial, que es el producto entre la velocidad de rotación y la distancia al centro de la fuente de calor [8].

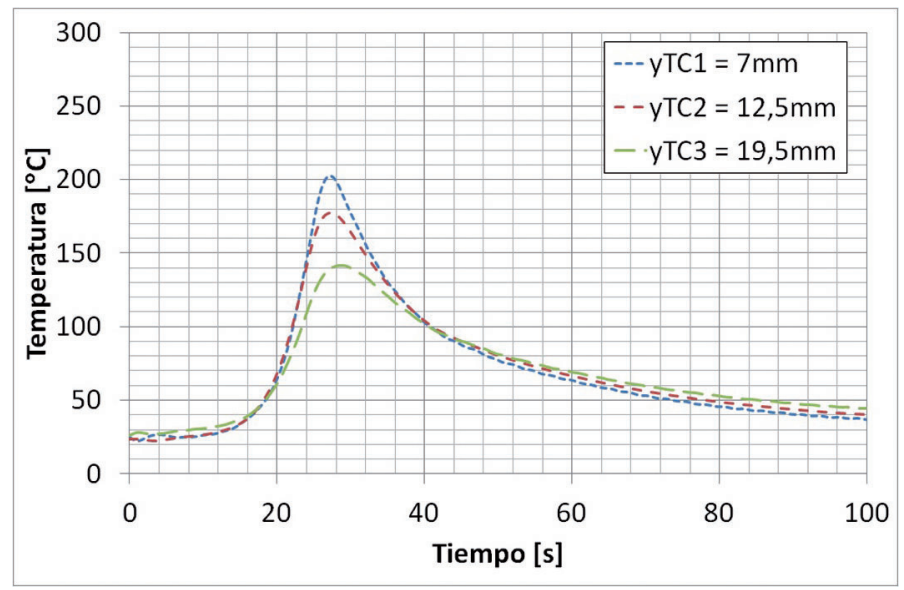

A
Por otra parte, se tuvieron en cuenta las pérdidas de calor hacia el ambiente, por conducción hacia la placa de apoyo y por convección en el resto de las superficies de contorno. El calor disipado por convección depende del coeficiente de convección entre el borde de la chapa y el ambiente, el cual se consideró de $20 \mathrm{~W} .\left(\mathrm{m}^{2} . \mathrm{K}\right)^{-1}$ [9]. Asimismo, se supuso que la transferencia de calor hacia la placa de apoyo es con un sólido lo suficientemente grande que no cambia su temperatura $\left(20^{\circ} \mathrm{C}\right)$. Este calor disipado por conducción depende del coeficiente de conducción, que si bien puede estimarse, es un parámetro desconocido que se determinó experimentalmente al calibrar el modelo de elementos finitos.

Finalmente, se obtuvo la potencia neta $(\mathrm{Q})$ necesaria para que la temperatura máxima calculada en la posición de la TC1 sea igual a la temperatura máxima medida por la misma.

\subsection{Caracterización Macroestructural y Determinación de Propiedades Mecánicas}

De cada probeta soldada se extrajo un corte transversal, el cual se preparó para observación macrográfica. Se analizaron las distintas zonas de las uniones soldadas, así como la presencia de discontinuidades asociadas a la soldadura. Sobre las mismas probetas metalográficas se determinaron perfiles de microdureza

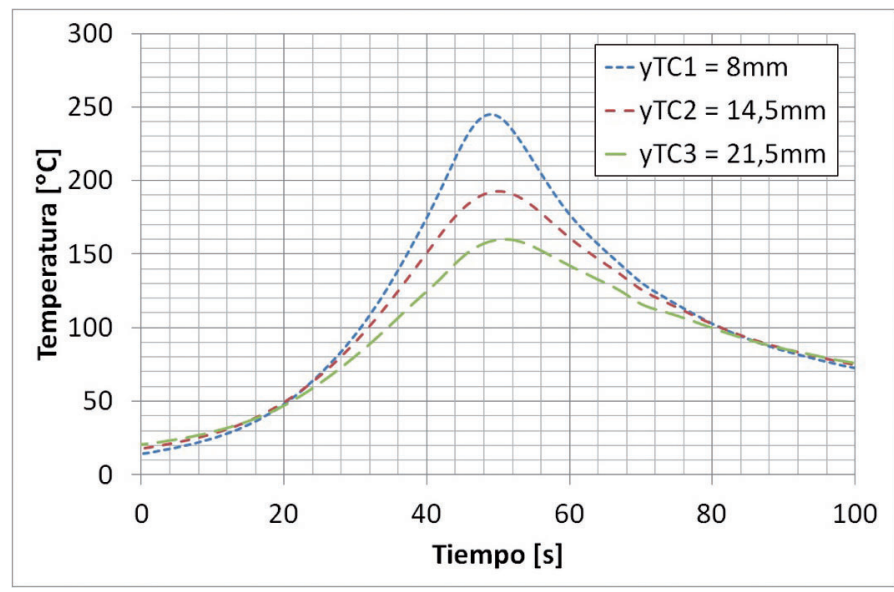

B

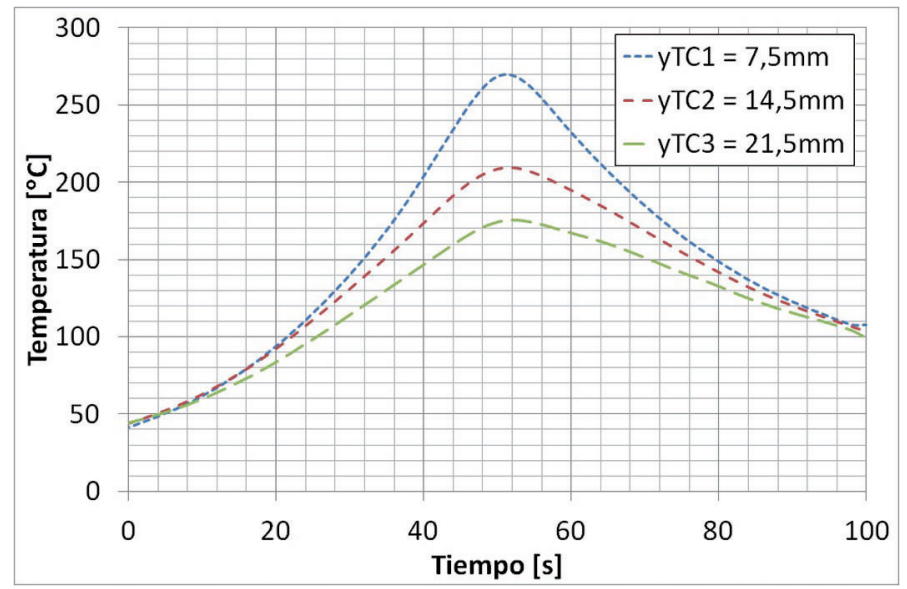

C

Figura 2. Ciclos térmicos adquiridos: A. Probeta 514-206, B. Probeta 514-73, C. Probeta 680-51 
Vickers a lo largo de la sección transversal sobre la línea media del espesor, a distintos tiempos luego de realizada la soldadura. Dichas mediciones se realizaron empleando una carga de 500 gf. En todas las macrografías y perfiles de microdureza el lado derecho corresponde al lado de avance.

Por otra parte, de cada probeta soldada se extrajeron y mecanizaron probetas de tracción, las cuales se ensayaron a distintos tiempos luego de realizada la soldadura, según norma ASTM E8M, obteniéndose la resistencia a la tracción. Posteriormente se analizaron las superficies de fractura, determinándose la ubicación de las mismas y su relación con la existencia de defectos.

Los tiempos evaluados para los perfiles de microdureza y ensayos de tracción variaron entre 1 y 900 días, aproximadamente.

\section{Resultados y Discusión}

\subsection{Ciclos Térmicos Adquiridos}

En la Figura 2 se muestran los ciclos térmicos adquiridos para las tres condiciones de soldadura analizadas, pudiéndose observar claramente como la temperatura máxima disminuyó con la distancia al centro del cordón de soldadura. Asimismo, puede verse como se modifican significativamente los ciclos térmicos experimentados por el material al variar las condiciones de soldadura.

En la Figura 3 se comparan los ciclos térmicos adquiridos por la termocupla más cercana al cordón de soldadura (TC1) para las tres condiciones de soldadura. En la misma se puede apreciar como la temperatura máxima aumentó significativamente con el aumento del número de Pitch, mientras que las velocidades de enfriamiento y calentamiento disminuyeron, motivo por el cual se puede considerar que el material estuvo sometido a mayores temperaturas por un tiempo mayor.

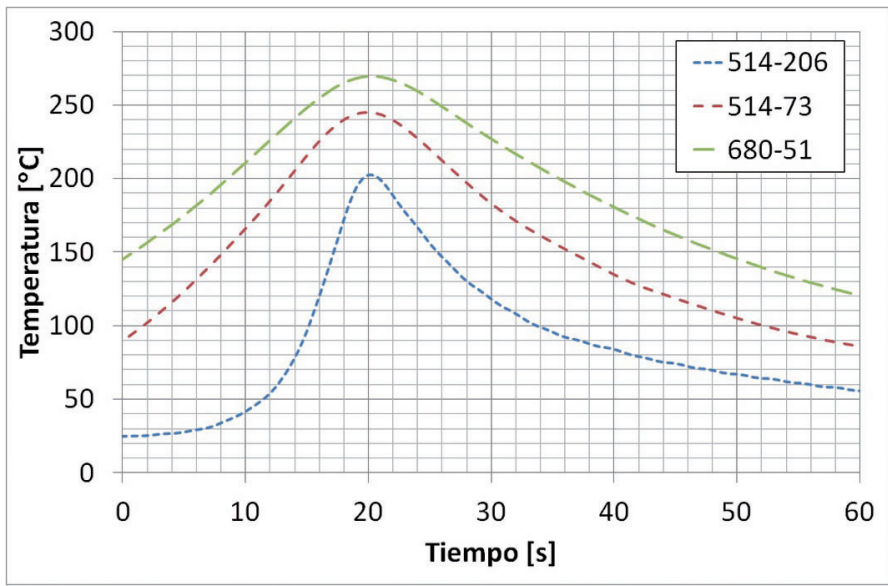

Figura 3. Comparación de los ciclos térmicos adquiridos por la TC1

En la Tabla 2 se muestran los valores característicos de los ciclos térmicos adquiridos. Como se explicó en la sección anterior, a partir de la posición y temperatura máxima correspondientes a la TC1 se obtuvieron mediante un modelo de FEM los valores netos de la potencia (Q) y del calor aportado $(\mathrm{H}=\mathrm{Q} / \mathrm{U})$.

Tabla 2. Valores característicos de los ciclos térmicos adquiridos

\begin{tabular}{|c|c|c|c|c|c|}
\hline Designación & $\begin{array}{c}\text { Pitch }=\mathrm{w} / \mathrm{U} \\
\left(\mathrm{rev} . \mathrm{mm}^{-1}\right)\end{array}$ & $\begin{array}{c}\text { Posición de } \\
\text { TC1 }(\mathrm{mm})\end{array}$ & $\begin{array}{c}\text { Temperatura Máxima } \\
\text { TC1 }\left({ }^{\circ} \mathrm{C}\right)\end{array}$ & Potencia $(\mathrm{W})$ & $\begin{array}{c}\text { Calor Aportado } \\
\left(\mathrm{J} . \mathrm{mm}^{-1}\right)\end{array}$ \\
\hline $514-206$ & 2,5 & 7 & 203 & 453 & 132 \\
\hline $514-73$ & 7,0 & 8 & 245 & 440 & 362 \\
\hline $680-51$ & 13,3 & 7,5 & 270 & 444 & 522 \\
\hline
\end{tabular}

Los valores de la tabla anterior muestran que la potencia se mantuvo prácticamente invariante para las tres condiciones de soldadura. Por otra parte, el calor aportado aumentó con el número de Pitch, presentando una tendencia lineal, como se puede observar en la Figura 4. Estos resultados son consistentes con lo reportado en trabajos anteriores para uniones soldadas similares [7].

\subsection{Caracterización Macroestructural}

En la Figura 5 se muestran las macrografías de los cortes transversales de las probetas soldadas, donde el lado derecho se corresponde con el lado de avance. En las mismas se pueden apreciar claramente las distintas zonas características de la soldadura por fricción-agitación y que para las tres condiciones de soldadura se obtuvo una penetración total.

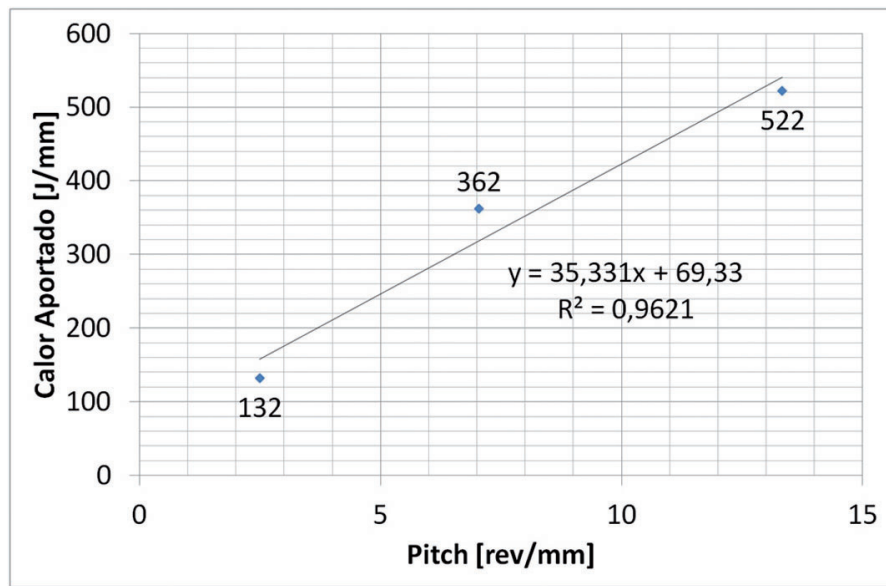

Figura 4. Calor Aportado calculado mediante FEM en función del número de Pitch 


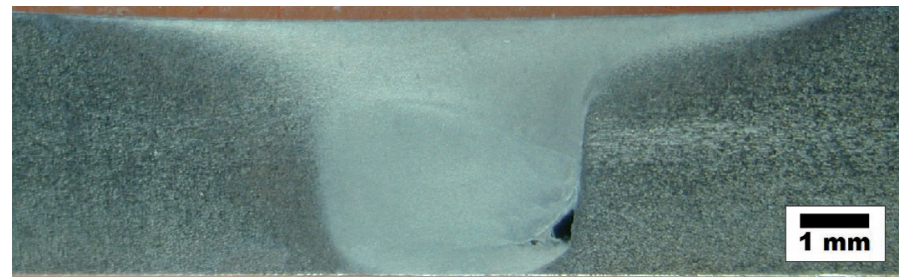

A

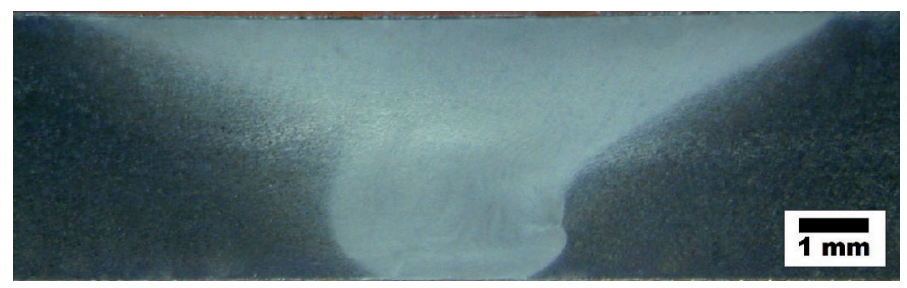

$\mathrm{B}$

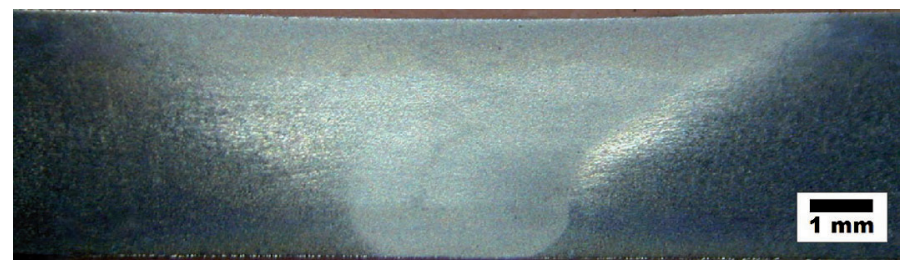

$\mathrm{C}$

Figura 5. Macrografías de las probetas soldadas: A. Probeta 514-206, B. Probeta 514-73, C. Probeta 680-51

Las probetas 514-73 y 680-51 mostraron un buen flujo plástico y ausencia de defectos macroscópicos. A diferencia de las anteriores, la probeta 514-206 presentó un defecto "túnel" en la interfase, entre la zona agitada (WN) y la zona termomecánicamente afectada (TMAZ) del lado de avance. Además, se observa que el tamaño de la zona agitada aumenta con el número de pitch, siendo para la condición 514-206 relativamente menor al de las otras dos probetas. Ambos resultados están relacionados con el menor calor aportado obtenido para esta condición de soldadura, el cual se analizó en la sub-sección anterior. Debido a este menor aporte térmico, se tuvo un flujo plástico de material más limitado, el cual resultó insuficiente para alcanzar el lado de avance desde el lado de retroceso por detrás de la herramienta, generando el defecto observado [10].

\subsection{Perfiles de Microdureza}

En la Figura 6 se muestran los perfiles de microdureza obtenidos a diferentes tiempos de envejecimiento para las tres condiciones de soldadura estudiadas. Para poder relacionar los valores de microdureza y las diferentes zonas de la unión, junto a los perfiles de microdureza se muestra una macrografía típica de un corte transversal de uniones soldadas por fricción-agitación del mismo material.

Los perfiles de microdureza medidos presentaron la forma en W característica de las uniones soldadas por fricción-agitación de aleaciones termoenvejecibles, con un ablandamiento en las zonas cercanas al cordón de soldadura y un valor de microdureza mínima en la zona afectada por el calor (HAZ) [1, 2]. Además, para las tres condiciones de soldadura analizadas, se observó un aumento considerable de la microdureza en las distintas zonas de la unión con el tiempo de envejecimiento, entre 1 y 900 días. Este comportamiento está relacionado con el envejecimiento natural que se produce a partir de realizada la soldadura y ha sido reportado anteriormente en la literatura $[4,5]$.

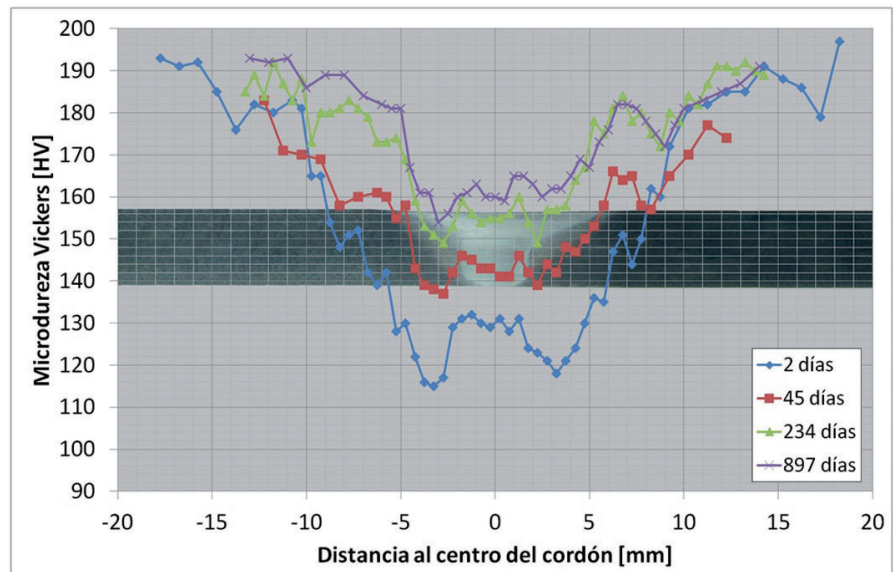

A

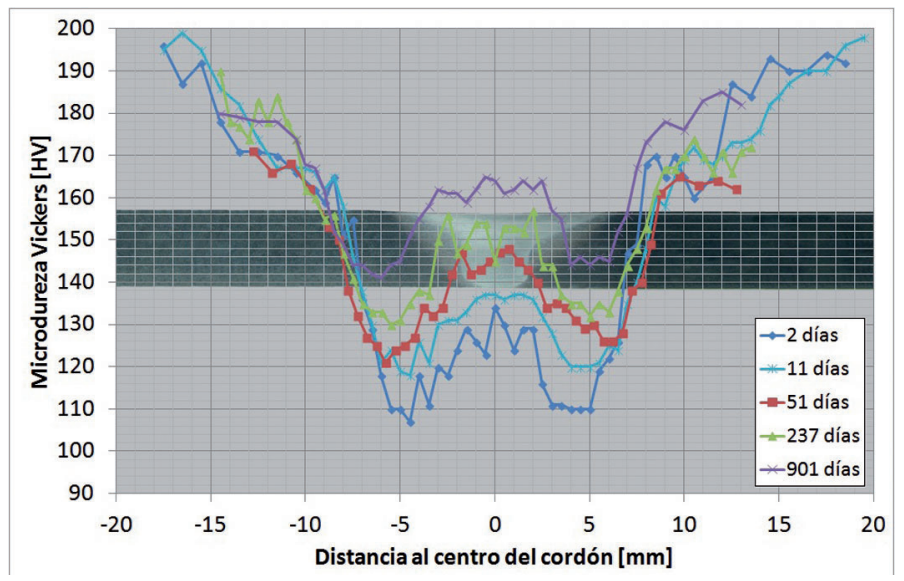

B

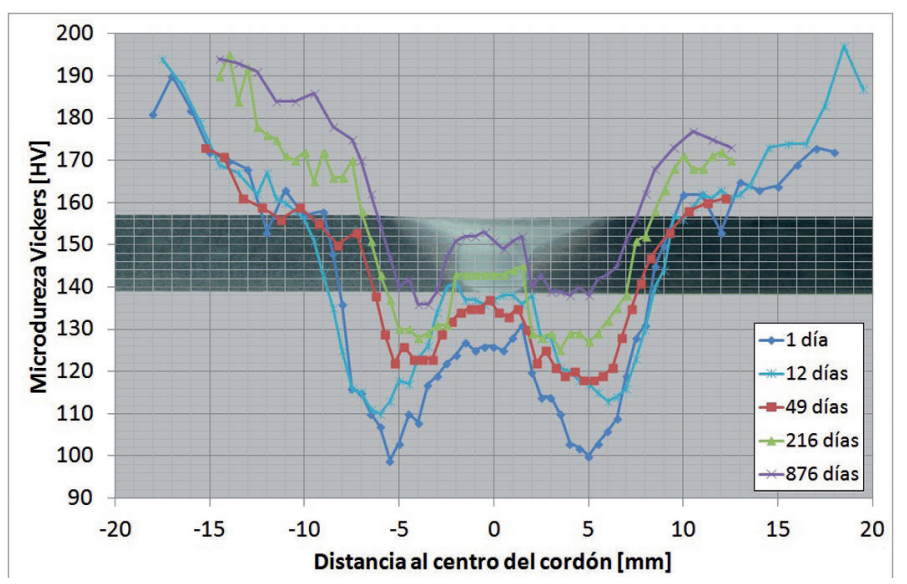

$\mathrm{C}$

Figura 6. Perfiles de microdureza para diferentes tiempos de envejecimiento de las probetas soldadas: A. Probeta 514-206, B. Probeta 514-73, C. Probeta 680-51 
Tabla 3. Valores característicos de los perfiles de microdureza para distintos tiempos de envejecimiento

\begin{tabular}{|c|c|c|c|}
\hline Designación & $\begin{array}{c}\text { Tiempo de } \\
\text { Envejecimiento } \\
\text { (días) }\end{array}$ & $\begin{array}{l}\text { Microdureza } \\
\text { Mínima (HV) }\end{array}$ & $\begin{array}{l}\text { Microdureza de la } \\
\text { Zona Agitada (HV) }\end{array}$ \\
\hline \multirow{4}{*}{ 514-206 } & 2 & 115 & 130 \\
\hline & 45 & 137 & 146 \\
\hline & 234 & 149 & 156 \\
\hline & 897 & 154 & 162 \\
\hline \multirow{5}{*}{$514-73$} & 2 & 107 & 128 \\
\hline & 11 & 118 & 136 \\
\hline & 51 & 121 & 145 \\
\hline & 237 & 130 & 151 \\
\hline & 901 & 141 & 162 \\
\hline \multirow{5}{*}{$680-51$} & 1 & 99 & 126 \\
\hline & 12 & 110 & 137 \\
\hline & 49 & 118 & 134 \\
\hline & 216 & 125 & 143 \\
\hline & 876 & 136 & 151 \\
\hline
\end{tabular}

Por otra parte, si se observa la Figura 6.A, se puede apreciar claramente que la forma en $\mathrm{W}$ de los perfiles de microdureza fue cada vez menos evidente a medida que aumentó el tiempo de envejecimiento, es decir, que la microdureza mínima $\left(\mathrm{HV}_{\min }\right)$ y la microdureza de la zona agitada $\left(\mathrm{HV}_{\mathrm{wN}}\right)$ fueron cada vez más similares.

Por último, si se comparan los perfiles de microdureza de diferentes condiciones de soldadura correspondientes a tiempos de envejecimiento similares, se puede observar que la microdureza en la zona afectada por el calor (HAZ) disminuyó con el aumento del aporte térmico, asociado a una mayor degradación térmica de la microestructura, consistentemente con lo reportado en la literatura [3]. El comportamiento observado puede asociarse a que el material estuvo sometido a mayores temperaturas por un tiempo mayor a medida que aumentó el número de pitch, resultando en un mayor sobreenvejecimiento y una mayor disolución de los precipitados en la zona afectada por el calor (HAZ) y una menor velocidad de enfriamiento, por lo que se produce una mayor disminución de la $\mathrm{HV}_{\min }[11,12]$.

Para continuar el análisis de la evolución temporal de los perfiles de microdureza, en la Tabla 3 se muestran valores característicos de los mismos, es decir, el valor de microdureza mínima y el valor promedio de microdureza de la zona agitada.

En la Figura 7 se grafica la $\mathrm{HV}_{\text {min }}$ y la $\mathrm{HV}_{\mathrm{wN}}$ en función del tiempo de envejecimiento para las tres condiciones de soldadura. Para poder comparar estos valores con la microdureza del material base, la misma se indicó con una línea de trazos horizontal, siendo el promedio de $193 \mathrm{HV}$.

En general, el estudio del problema de la influencia del procedimiento de soldadura por FSW sobre la degradación de las propiedades mecánicas por la soldadura y su evolución temporal en aleaciones termoenvejecibles, puede dividirse en dos efectos. Por un lado, el efecto sobre la disminución de la dureza luego de

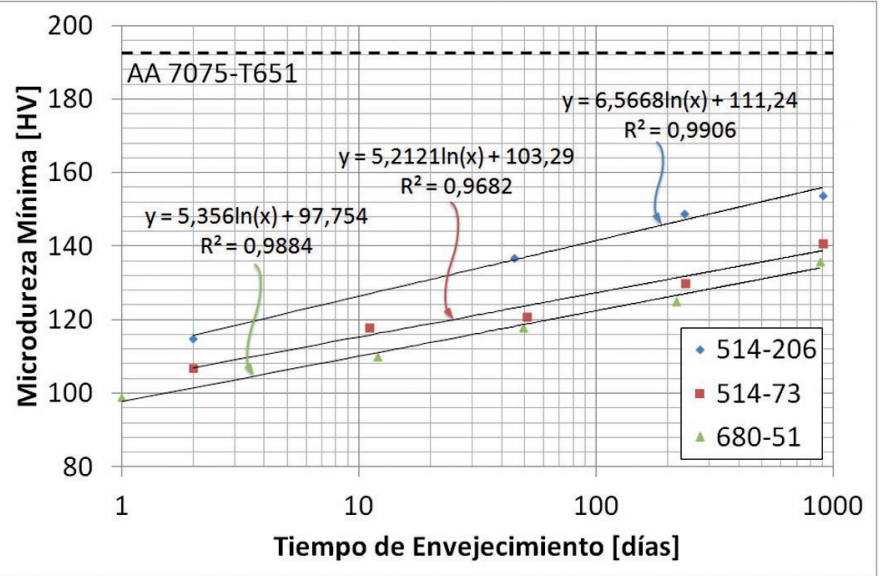

la soldadura y por otro sobre su evolución con el tiempo.

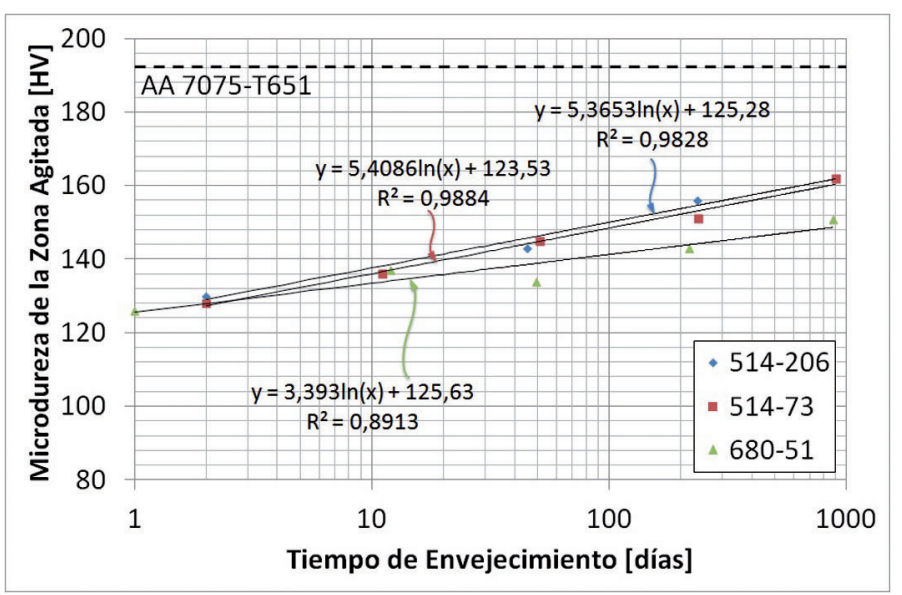

A

$\mathrm{B}$

Figura 7. Microdureza Mínima (A) y de la Zona Agitada (B) en función del tiempo de envejecimiento 
En los gráficos de la Figura 7 se puede observar claramente el aumento de la $\mathrm{HV}_{\text {min }}$ y de la $\mathrm{HV}_{\mathrm{WN}}$ con el tiempo de envejecimiento, presentando una tendencia logarítmica con un buen grado de ajuste en todos los casos. Por este motivo, el eje correspondiente al tiempo de envejecimiento se muestra en escala logarítmica y las tendencias logarítmicas se observan como líneas rectas.

Por otra parte, existen algunas diferencias en la evolución temporal de estos valores característicos de los perfiles de microdureza. En la Figura 7.A se puede apreciar que las líneas de tendencia de la $\mathrm{HV}_{\min }$ no se cruzan entre si y que son aproximadamente paralelas, aunque la pendiente de la curva correspondiente a la probeta 514-206 es mayor a las otras. Además, en esta figura se puede observar claramente que la $\mathrm{HV}_{\text {min }}$ para las diferentes condiciones de soldadura correspondientes a tiempos de envejecimiento similares disminuyó con el calor aportado, lo que está relacionado con el análisis de la Figura 6. En cambio, las líneas de tendencia de la Figura 7.B correspondientes a la $\mathrm{HV}_{\mathrm{wN}}$ se tiene que parten de un mismo valor para 1 día luego de soldadas, siendo en este caso la pendiente de la curva correspondiente a la probeta 680-51 menor a las otras.

Para analizar la evolución temporal de la microdureza es necesario partir de una condición inicial. Para ello podría utilizarse la microdureza con 1 día de envejecimiento, que si bien podría obtenerse de perfiles de microdureza medidos a ese tiempo después de realizada la unión soldada, se determinaron a partir de las constantes obtenidas de las tendencias logarítmicas de la Figura 7. De esta manera, en la Figura 8 se grafica la microdureza a 1 día de envejecimiento correspondiente a la $\mathrm{HV}_{\text {min }}$ y a la $\mathrm{HV}_{\mathrm{WN}}$ para las tres condiciones de soldadura

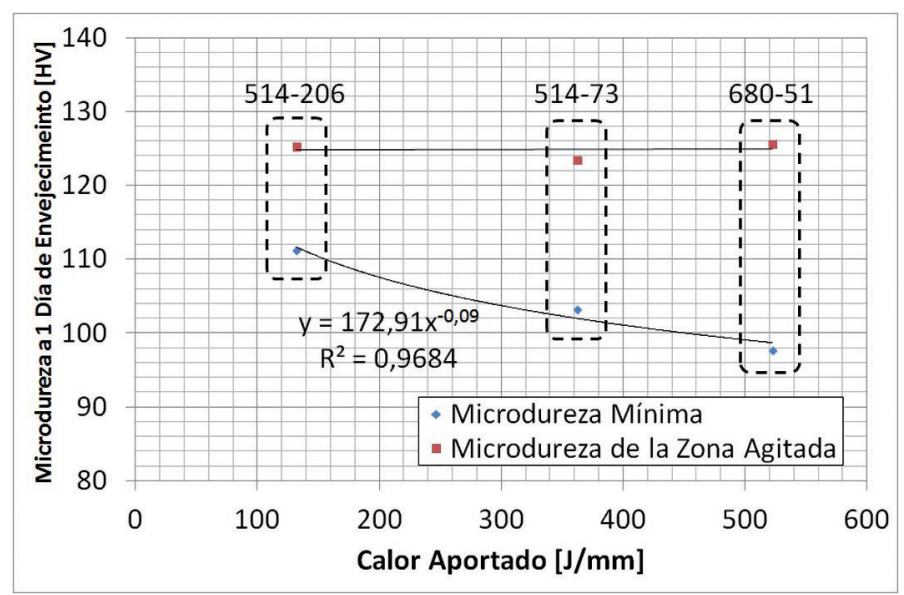

analizadas, en función del calor aportado.

Figura 8. Microdureza a 1 día de envejecimiento para las tres condiciones de soldadura analizadas

En relación a lo que se dijo anteriormente, en la Figura 8 se puede apreciar que la $\mathrm{HV}_{\min }$ a 1 día de envejecimiento disminuyó con el calor aportado, presentando una tendencia potencial. Esta relación entre ambos parámetros se ha reportado en trabajos anteriores para uniones soldadas similares [11]. Los valores alcanzados representan entre un 51 y $60 \%$ del valor de dureza original. Sin embargo, la $\mathrm{HV}_{\mathrm{wN}}$ se vería afectada por otros factores adicionales, asociados con la deformación plástica sufrida durante la soldadura, como la velocidad de deformación, que afectarán aspectos como la recristalización dinámica, definiendo entre otros el tamaño de grano resultante. En esta zona, la microdureza a 1 día de envejecimiento se mantuvo prácticamente invariante para las tres condiciones de soldadura en el orden de los $125 \mathrm{HV}$.

Finalmente, cuanto mayor sea la diferencia entre la $\mathrm{HV}_{\text {min }}$ y la $\mathrm{HV}_{\mathrm{WN}}$, más notoria es la forma en $\mathrm{W}$ del perfil de microdureza. En este sentido se observa que dicha diferencia entre ambos valores de dureza aumentó con el calor aportado, siendo la condición 514-206 la que mostró menor diferencia, consistentemente con una forma en $\mathrm{W}$ del perfil de microdureza menos acentuada, lo que pudo apreciarse en la Figura 6.

Una vez determinado el valor de microdureza a 1 día de envejecimiento como condición inicial, la evolución temporal queda definida por la tasa de endurecimiento. Sin embargo, la tasa de endurecimiento determinada como incrementos de dureza sobre incrementos temporales disminuye monótonamente con el tiempo. Esto se debe a que las tendencias son logarítmicas. Por lo tanto, en este trabajo se propone la definición de tasa de endurecimiento logarítmica, que es el factor que multiplica al logaritmo natural del tiempo de envejecimiento expresado en días en las tendencias logarítmicas de la Figura 7 y que tiene unidades de microdureza. La ventaja de utilizar la tasa de endurecimiento logarítmica es que es un valor constante durante toda la evolución temporal, siendo la pendiente de las líneas de tendencia de los gráficos semi-logarítmicos de dicha figura. De esta manera, en la Figura 9 se grafica la tasa de endurecimiento logarítmica correspondiente a la $\mathrm{HV}_{\text {min }}$ y a la $\mathrm{HV}_{\mathrm{wN}}$ para las tres condiciones de soldadura analizadas. Utilizando el mismo criterio que en la Figura 8, este parámetro se muestra en función

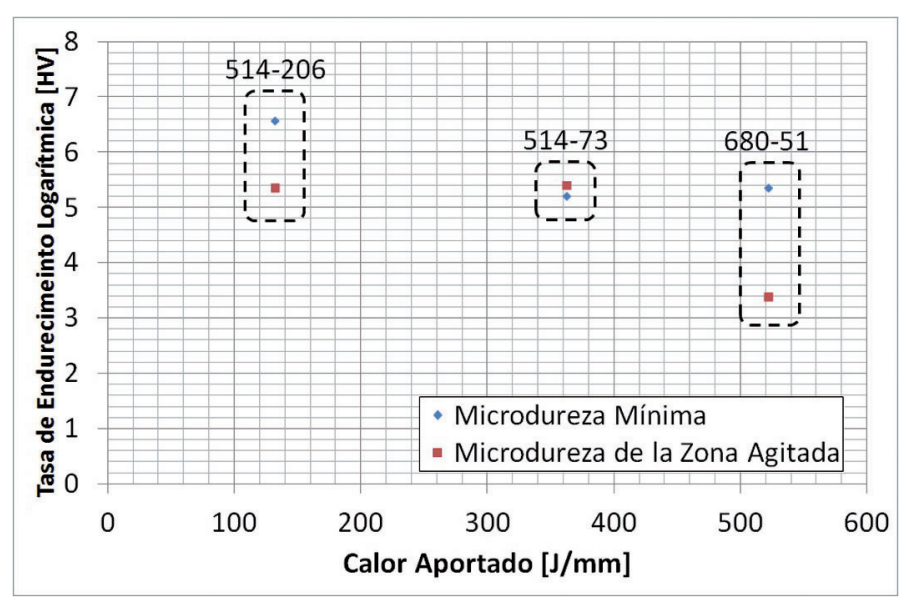

del calor aportado.

Figura 9. Tasa de endurecimiento logarítmica para las tres condiciones de soldadura analizadas

En primer lugar, se puede observar que la mayoría de las tasas de endurecimiento logarítmicas fueron del orden de los 5,3 HV. Sin embargo, para la probeta 514-206, correspondiente a la condición de soldadura con el menor aporte térmico, este parámetro en la zona de $\mathrm{HV}_{\text {min }}$ fue mayor al resto, siendo de 6,6 


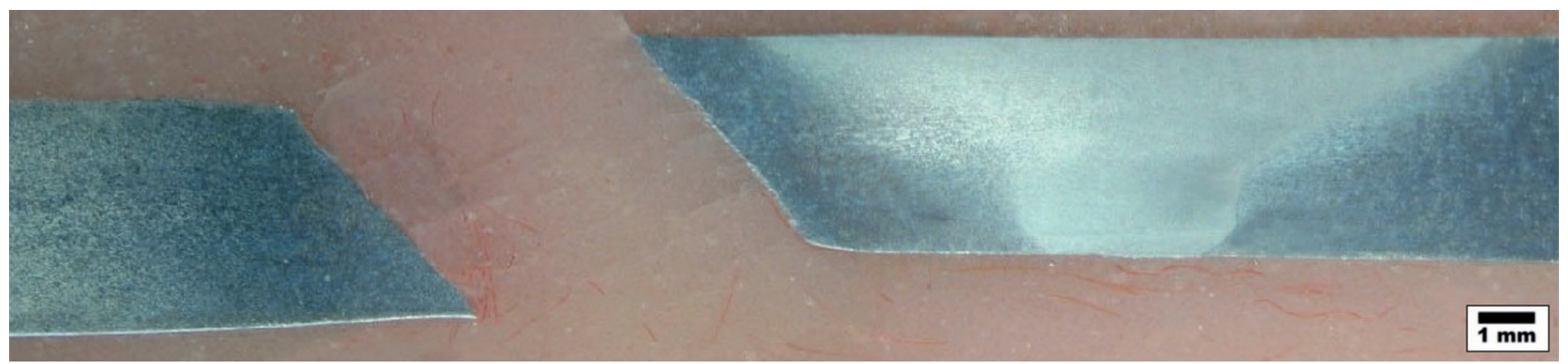

Figura 10. Ubicación de la fractura en el ensayo de tracción de la probeta 680-51

HV. Si además se tiene en cuenta que la $\mathrm{HV}_{\text {min }}$ correspondiente a 1 día de envejecimiento fue mayor para esta condición de soldadura, este resultado indica que la probeta 514-206 no solo es la que sufrió una menor degradación de su microestructura, sino que además es la que experimentó un mayor aumento en la microdureza.

Por otra parte, para la probeta 680-51, que fue la única unión soldada con una velocidad de rotación de $680 \mathrm{rpm}$, la tasa de endurecimiento logarítmica en la zona agitada fue menor al resto, siendo de 3,4 HV. De esta manera, para las condiciones 514-206 y 680-51 la tasa de endurecimiento logarítmica fue mayor para la $\mathrm{HV}_{\text {min }}$ que para la $\mathrm{HV}_{\mathrm{wN}}$, como se puede apreciar en la Figura 9. Por lo tanto, para estas condiciones ambos valores característicos de microdureza fueron cada vez más similares, generando perfiles de microdureza con una forma en $\mathrm{W}$ cada vez menos marcada, como se puede apreciar en la Figura 6.A y Figura 6.C. Además, como para la probeta 514206 la diferencia entre ambos valores de microdureza a 1 día de envejecimiento fue menor, el efecto de la uniformización del perfil de microdureza fue más importante para esta condición de soldadura.

Un caso particular se da para la probeta 514-73, donde la tasa de endurecimiento logarítmico es prácticamente igual para la $\mathrm{HV}_{\text {min }}$ y la $\mathrm{HV}_{\mathrm{wN}}$. Esto produjo que la evolución temporal de la microdureza fuera más uniforme, por lo que la diferencia que se observó en los valores característicos de microdureza a 1 día de envejecimiento en la Figura 8 se mantuvo en el tiempo y no se modificó la forma del perfil de microdureza, como puede observarse en la Figura 6.B.

\subsection{Resistencia a la Tracción}

La mayoría de las probetas de tracción obtenidas de las uniones soldadas 514-206 y 514-73 presentaron una fractura que se ubicó en la zona agitada (WN) y que estuvo relacionada con algún defecto, como el defecto túnel observado en la Figura 5.A o la continuidad de la línea de óxidos presente en la zona agitada. Ambos son defectos característicos de la soldadura por fricción-agitación, relacionados con la falta de calor aportado y un flujo plástico de material insuficiente [3].

Si la zona agitada (WN) se encuentra libre de defectos, la resistencia a la tracción de la unión soldada estaría asociada a la $\mathrm{HV}_{\text {min }}$ y la fractura se ubicaría en la posición de la misma en la zona afectada por el calor (HAZ) [3]. En este sentido, para la condición 680-51, que es la correspondiente al mayor calor aportado, las fracturas de las probetas de tracción se ubicaron en la posición de la $\mathrm{HV}_{\min }$, como se puede apreciar en la macrografía de la Figura 10.

De esta manera, como la resistencia a la tracción de la probeta soldada 680-51 está asociada a la $\mathrm{HV}_{\text {min }}$, sobre esta condición de soldadura se analizará la evolución temporal de la resistencia la tracción. En la Tabla 4 se presentan los valores de resistencia a la tracción y de eficiencia de la unión soldada de la probeta 680-51 para distintos tiempos de envejecimiento. Este último parámetro se calculó considerando que la resistencia a la tracción del material base obtenida fue de $576 \mathrm{MPa}$.

Tabla 4. Resistencia a la tracción y eficiencia de la probeta 680-51 para distintos tiempos de envejecimiento

\begin{tabular}{|c|c|c|}
\hline $\begin{array}{c}\text { Tiempo de } \\
\text { Envejecimiento } \\
\text { (días) }\end{array}$ & $\begin{array}{c}\text { Resistencia a la } \\
\text { Tracción (MPa) }\end{array}$ & $\begin{array}{c}\text { Eficiencia } \\
(\%)\end{array}$ \\
\hline 1 & 373 & 65 \\
\hline 12 & 414 & 72 \\
\hline 63 & 428 & 75 \\
\hline 99 & 447 & 78 \\
\hline 124 & 442 & 77 \\
\hline 168 & 452 & 78 \\
\hline 529 & 463 & 80 \\
\hline
\end{tabular}

La resistencia a la tracción a 1 día de envejecimiento fue de $373 \mathrm{MPa}$, mientras que a los 529 días después de realizada la unión soldada la resistencia a la tracción fue de $463 \mathrm{MPa}$. Para los mismos tiempos de envejecimiento, la eficiencia de la unión soldada aumentó del $65 \%$ al $80 \%$. De esta manera, a los 529 días se obtuvo un incremento en la resistencia a la tracción de 90 $\mathrm{MPa}$, es decir, una resistencia a la tracción un $24 \%$ mayor a la correspondiente a 1 día de envejecimiento.

Para continuar el análisis de la evolución temporal de la resistencia a la tracción de la probeta 680-51, en la Figura 11 se grafican los valores de la misma en función del tiempo de envejecimiento. De manera similar que en la Figura 7, se indicó la resistencia a la tracción del material base con una línea de trazos horizontal.

Al igual que los valores de $\mathrm{HV}_{\text {min }}$, la resistencia a la tracción aumentó con el tiempo de envejecimiento, presentando una 


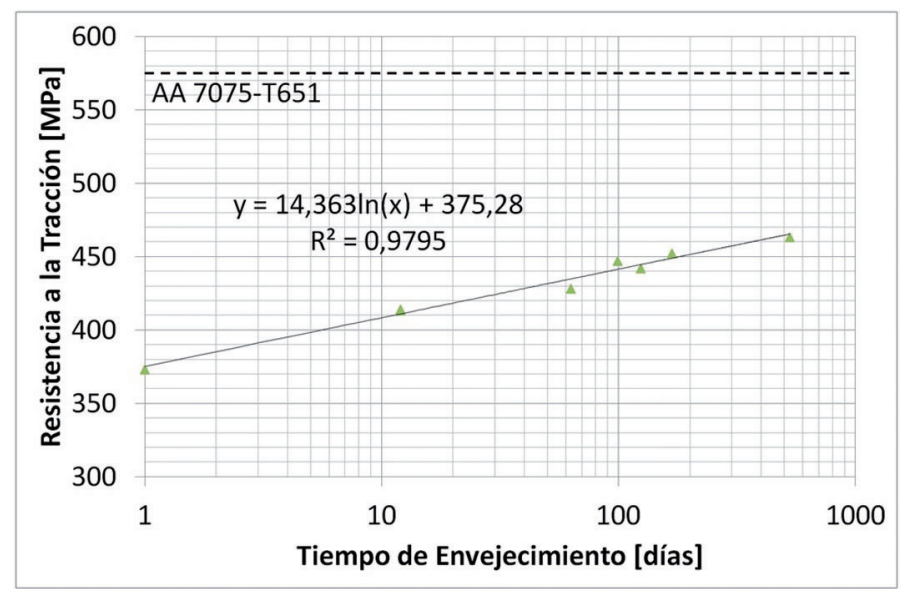

Figura 11. Resistencia a la tracción de la probeta 680-51 en función del tiempo de envejecimiento

tendencia logarítmica con un buen grado de ajuste. Por lo tanto, este resultado indica que si la junta no presenta defectos en la zona agitada, la resistencia a la tracción queda determinada por la $\mathrm{HV}_{\text {min }}$ y que ambos parámetros aumentan con el tiempo de envejecimiento de manera similar, siguiendo una ley logarítmica.

\section{Conclusiones}

Las propiedades mecánicas de las uniones soldadas por FSW en AA7075-T651 se ven afectada por las condiciones de soldadura y por el tiempo transcurrido luego de realizada la soldadura.

Los perfiles de microdureza de las uniones soldadas presentan una forma de $\mathrm{W}$, identificándose como valores característicos la microdureza mínima $\left(\mathrm{HV}_{\min }\right)$ y la microdureza de la zona agitada $\left(\mathrm{HV}_{\mathrm{wN}}\right)$.

La microdureza mínima luego de 1 día de realizada la soldadura disminuyó con el calor aportado, presentando una tendencia potencial, alcanzando valores de entre un 51 y $60 \%$ de la dureza del material base. Asimismo, la microdureza de la zona agitada fue independiente del procedimiento de soldadura, alcanzado en todos los casos $125 \mathrm{HV}$.

Debido al envejecimiento natural que se produce a partir de realizada la soldadura, se observó un aumento considerable de la microdureza en las distintas zonas de la unión con el tiempo. En particular, la microdureza mínima y la microdureza de la zona agitada aumentaron con el tiempo de envejecimiento siguiendo una ley logarítmica. Por otra parte, se introdujo la definición de tasa de endurecimiento logarítmica, la cual fue para la mayoría de los casos del orden de los 5,3 HV, tanto para la zona de microdureza mínima como para la zona agitada.

En particular, para la probeta 514-206, correspondiente a la condición de soldadura con el menor aporte térmico, la tasa de endurecimiento logarítmica en la zona de microdureza mínima fue mayor al resto, por lo que no solo es la condición que sufrió una menor degradación de su microestructura, sino que además es la que experimentó un mayor aumento en la microdureza. Además, como la tasa de endurecimiento logarítmica fue mayor para la microdureza mínima que para la microdureza de la zona agitada, ambos valores característicos de microdureza fueron cada vez más similares, por lo que la forma en $\mathrm{W}$ del perfil de microdureza característica de las uniones soldadas por fricciónagitación de aleaciones termoenvejecibles fue cada vez menos notable.

Finalmente, para la probeta 680-51, que es la correspondiente al mayor calor aportado y velocidad de rotación, las fracturas de las probetas de tracción se ubicaron en la posición de la microdureza mínima, lo que indica que esta condición presentó un bajo nivel de defectos en la zona agitada y que la resistencia a la tracción quedó determinada por la microdureza mínima. De esta manera, también se observó un aumento de la resistencia a la tracción con el tiempo de envejecimiento siguiendo una ley logarítmica, obteniéndose en el período de 1 a 529 días de envejecimiento un aumento de la eficiencia de junta del $65 \%$ al $80 \%$.

\section{Agradecimientos}

Los autores desean agradecer a INTI-Mecánica y a la Universidad de Buenos Aires por el financiamiento y la utilización de sus instalaciones para el desarrollo del presente trabajo. Así también al personal de INTI-Mecánica y del Laboratorio de Materiales y Estructuras de FIUBA por la asistencia brindada.

\section{Referencias Bibliográficas}

[1] MISHRA, R.S.; MA, Z.Y. Friction stir welding and processing. Materials Science and Engineering R, v.50, p.1-78, 2005.

[2] NANDAN, R.; DEBROY, T.; BHADESHIA, H.K.D.H. Recent advances in friction-stir welding - Process, weldment structure and properties, Progress in Materials Science, v.53, p.980-1023, 2008.

[3] MISHRA, R.S.; MAHONEY, M.W. Friction Stir Welding and Processing. 1.ed. Ohio: ASM Internacional, 2007. 352 p.

[4] LINTON, V.M.; RIPLEY, M.I. Influence of time on residual stresses in friction stir welds in agehardenable $7 \mathrm{xxx}$ aluminium alloys, Acta Materialia, v.56, p.4319-4327, 2008.

[5] FUllER, C.B.; MAHONEY, M.W.; CALABRESE, M.; MICONA, L. Evolution of microstructure and mechanical properties in naturally aged 7050 and $7075 \mathrm{Al}$ friction stir welds, Materials Science and Engineering A, v.527, p.2233-2240, 2010.

[6] ASM INTERNACIONAL. ASM Handbook: Volume 2, Properties and Selection: Nonferrous Alloys and SpecialPurpose Materials. 2.ed. Ohio: ASM Internacional, 1992.

[7] TUFARO, L.N.; SVOBODA H.G. Estudio de los Ciclos Térmicos Adquiridos durante FSW mediante Modelos Analíticos y Numéricos. In: Congresso Nacional de Soldagem, 38º 2012, Ouro Preto. Anais CONSOLDA 2012.

[8] SCHMIDT, H. B. y HATTEL, J. H. Thermal modelling of friction stir welding. Scripta Materialia, v. 58, p. 332-337, 2008. [9] MILLS, A.F. Transferencia de Calor. 1.ed. México: Irwin, $1995.960 \mathrm{p}$.

[10] RAJAKUMAR, S.; MURALIDHARAN, C.; BALASUBRAMANIAN, V. Influence of friction stir welding process and 
tool parameters on strength properties of AA7075-T6 aluminium alloy joints, Materials and Design, v.32, p.535-549, 2011.

[11] TUFARO, L.N.; SVOBODA H.G. Efecto del procedimiento de soldadura por FSW en uniones de AA 7075-T651. In: Congreso Nacional de Materiales, $12^{\circ}$, 2012, Alicante. Anales IBEROMAT 2012.

[12] PASTOR, A; SVOBODA, H.G. Evolución temporal de la zona afectada por el calor en soldaduras por fricción agitación de AA7075-T651. In: Congreso Internacional en Ciencia y Tecnología de Metalurgia y Materiales, 13º, 2013, Puerto Iguazú. Anales SAM-CONAMET 2013. 